\title{
Cefalea nocturna y miedo a no despertar
}

\author{
M. Espinosa Arévalo ${ }^{a}$,A. Pato Pato ${ }^{b}, M B$. Fernández Álvarez \\ aPediatra, CS. Sárdoma, Vigo. España. \\ ${ }^{b}$ Neurología, Hospital POVISA, Vigo. España. \\ 'MIR-MFyC, CS SárdomalHospital POVISA,Vigo. España.
}

Fecha de publicación en Internet: 16 de noviembre de 2011

\section{Resumen}

Los quistes de la glándula pineal, habitualmente asintomáticos, suelen ser un hallazgo incidental en pruebas de imagen realizadas por otro motivo. Se han relacionado ocasionalmente con diversos síntomas como cefalea, crisis epilépticas o trastornos psíquicos, siendo el factor más importante para que se conviertan en sintomáticos su tamaño o la presencia de un sangrado intraquístico.

Presentamos el caso de una mujer de 14 años de edad con quiste en la glándula pineal de pequeño tamaño y sangrado en su interior que consulta por cambios en las características de su cefalea habitual y manifestaciones psicoafectivas.

Palabras clave: Quiste pineal. Cefalea.

Nocturnal headache and fear of not awakening

Abstract

Pineal gland cysts, frequently asymptomatic, are usually an incidental finding in imaging performed for another reason. They have occasionally been associated to various symptoms such as headache, seizures or mental disorders, being its size or the presence of intracystic bleeding the most important risk factors for becoming symptomatic.

Key words: Pineal cyst. Headache.

\section{Introducción}

La región pineal es un área anatómica que incluye, además de la glándula pineal, el receso posterior del III ventrículo, diversas estructuras cerebrales (lámina tectal, cuerpo calloso, tronco cerebral y tálamo), las cisternas subaracnoideas (cuadrigeminal, ambiens, velum interpo- situm), el acueducto de Silvio, la encrucijada dural falcotentorial y estructuras vasculares arteriales (cerebrales posteriores, coroideas anteriores) y venosas (cerebrales internas, vena de Galeno).

El epitálamo (glándula pineal) es una glándula de secreción interna de forma ovoidea, aplanada, de unos $5 \mathrm{~mm}$ de

Belén Fernández Álvarez, belenorense@gmail.com

Los autores declaran no presentar conflictos de intereses en relación con la preparación y publicación de este artículo. 
diámetro, que descansa sobre la lámina cuadrigémina y segrega la hormona melatonina (relacionada con la regulación de los ciclos de vigilia y sueño), y se puede considerar parte de las vías visuales.

Dentro de las masas de la región pineal, debemos hacer un diagnóstico diferencial entre diversos tumores, sobre todo gliomas y pineoblastomas, metástasis y otros procesos no tumorales, como los quistes.

Los quistes de la glándula pineal suelen ser un hallazgo incidental en pruebas de imagen realizadas por otro motivo. Habitualmente pequeños y asintomáticos, la edad media de aparición son los 28 años y se han relacionado con la aparición de dolor de cabeza (un 50\% en forma de migrañas, según Seifer et al. ${ }^{1}$ ). El factor más importante para que se conviertan en sintomáticos parece ser su tamaño, sobre todo si este supera los $2 \mathrm{~cm}$ de diámetro, o la denominada apoplejía pineal, un sangrado intraquístico, de origen incierto y de muy infrecuente aparición ${ }^{2}$. Por otro lado, también se ha descrito la asociación entre los quistes pineales y las crisis epilépticas con manifestaciones psicoafectivas ${ }^{3}$.

\section{Caso clínico}

Mujer de 14 años intervenida de astrocitoma de bajo grado en la región frontal derecha a los tres años de edad (sin secuelas) y diagnosticada de migraña sin aura a los seis años, por lo que permanece en seguimiento en consulta. Refiere empeoramiento de sus crisis de cefalea en el último mes, siendo ahora de aparición diaria y predominio nocturno, con mayor duración e intensidad y pobre respuesta al tratamiento con antiinflamatorios no esteroideos que tomaba habitualmente. Coincidiendo con los episodios describe una sensación de desmayo y un intenso miedo a morir, de forma similar a los síntomas que tenía cuando se diagnosticó el astrocitoma. Como antecedentes familiares destaca madre diagnosticada de síndrome ansioso, distimia y cefalea por abuso de analgésicos.

La exploración neurológica fue normal. Se solicita resonancia magnética (RM) craneal, que objetiva cambios posquirúrgicos a nivel frontal derecho sin evidencia de recidiva local del tumor y un quiste pineal (ya conocido) de $15 \mathrm{~mm}$ que aumentó de tamaño respecto a los controles anteriores (11 mm), con presencia de sangrado en su interior. Se realiza analítica con Gnrh, FSH, LH, PRL, GH, ACTH y hormona de crecimiento (HCG) y un electroencefalograma, sin demostrar alteraciones.

Se decidió tratamiento sintomático de la cefalea y nuevo control radiológico en 
tres meses, donde se evidenció resolución del sangrado coincidiendo con la desaparición de los síntomas.

\section{Discusión}

La etiopatogenia de los quistes pineales ha sido motivo frecuente de controversia. Su aparición habitual tras el inicio de la pubertad hace suponer una posible relación con la involución de la glándula pineal ${ }^{4}$, sin embargo, pueden observarse a cualquier edad, desde la infancia hasta la vida adulta. Se han sugerido otras hipótesis para intentar explicar su origen, desde un fallo de crecimiento de las células pineales hasta una posible degeneración glial debida a una isquemia local5.

Habitualmente, los quistes pineales suelen ser de pequeño tamaño y asintomáticos, y su diagnóstico suele ser incidental. Los síntomas suelen aparecer cuando el quiste mide $>2 \mathrm{~cm}$, o cuando se produce un sangrado en su interior ${ }^{2}$. La clínica depende sobre todo de la producción de hipertensión intracraneal, pudiendo aparecer cefalea, parálisis de la mirada, déficit sensitivos, motores o cerebelosos, e incluso el coma o la muerte 4 . Algunos autores consideran que un quiste pineal puede producir síntomas aislados, sobre todo cefaleas, sin que se cumpla ninguno de los criterios mencionados ${ }^{1}$ (> $2 \mathrm{~cm}$, sangrado). En su etiopatogenia se ha considerado la disminución de la producción de melatonina por estos quistes, que puede disminuir el umbral del dolor y empeorar una cefalea preexistente o aparecer de novo ${ }^{1}$. Se han descrito también varios casos de empeoramiento de las cefaleas por sangrado intraquístico, en quistes que hasta ese momento no se habían relacionado directamente con la clínica ${ }^{2,6}$. En cuanto a su trasformación hemorrágica, se ha asociado en ocasiones a un traumatismo craneal previo, a una posible influencia hormonal o incluso al propio crecimiento del quiste.

Por otro lado, Grippo J et al. ${ }^{3}$ relacionan el sistema límbico con crisis epilépticas parciales con manifestaciones psicoafectivas, estableciendo un continuo entre la disminución de la melatonina en pacientes con quistes pineales y la aparición de episodios epilépticos nocturnos y trastornos emocionales.

En nuestro caso, la paciente presentaba cambios en las características de su cefalea habitual, de predominio nocturno, aunque sin clínica de hipertensión craneal, acompañada de sensación de desmayo y miedo a morir de aparición nocturna, que podría tener relación con crisis parciales simples con clínica psíquica o bien tratarse de un síntoma aislado. Aunque la tomografía axial computari- 
zada cerebral podría objetivar una posible hidrocefalia secundaria, el diagnóstico del quiste debe realizarse por RM, donde resulta característico el nivel líquido causado por la acumulación de los productos hemáticos cuando se produce un sangrado agudo². Su diagnóstico definitivo se realiza por biopsia.

La conducta a seguir con los quistes pineales es el control de su tamaño y morfología mediante $\mathrm{RM}$, y tratamiento únicamente sintomático, salvo que sean de gran tamaño (sobre todo si es mayor de $1 \mathrm{~cm}$ ), cuando se comprueba la existencia de hidrocefalia o cuando comien- zan a empeorar o producir síntomas incapacitantes ${ }^{7}$, momento en que se recomienda tratamiento quirúrgico. En nuestro caso se decidió tratamiento sintomático y nuevo control radiológico en tres meses, donde se evidenció resolución del sangrado coincidiendo con la desaparición de los síntomas.

La intervención se puede realizar por vía convencional ${ }^{8}$ o bien por cirugía endoscópica ${ }^{9,10}$, intentando la resección más completa posible de la glándula. Aunque si ya se ha producido sangrado intraquístico se considera la resección como la mejor alternativa².

\section{Bibliografía}

1. Seifert CL, Woeller A, Valet M, Zimmer C, Berthele $A$, Tölle $T$ et al. Headaches and pineal cyst: a case-control study. Headache. 2008;48: 448-52.

2. Patel AJ, Fuller GN, Wildrick DM, Sawaya R. Pineal cyst apoplexy: case report and review of the literature. Neurosurgery. 2005;57:1066.

3. Grippo J, Corral MS, Grippo T. Sistema límbico y crisis parciales con manifestaciones psicoafectivas. Rev Neurol. 2001;33:145-8.

4. Klein P, Rubinstein LJ. Benign symptomatic glial cysts of the pineal gland: a report of seven cases and review of the literature. J Neurol Neurosurg Psychiatry. 1989;52:991-5.

5. Gómez-Argüelles JM, Mata $P$, Bermejo $P E$, Anciones B. Agravamiento de un cuadro migrañoso por apoplejía de un quiste pineal gigante. Rev Neurol. 2009;48:17-9.
6. Musolino A, Cambria S, Rizzo G, Cambria M Symptomatic cysts of the pineal gland: stereotactic diagnosis and treatment of two cases and review of the literature. Neurosurgery. 1993;32:31520.

7. Vallée $B$, Meriot $P$, Person $H$, Rodríguez $V$ Sidhamed S, Dam Hieu P. Benign glial cysts of the pineal region. Neurochirurgie. 1997;43:299-302.

8. Hancq S, De Witte O, Brotchi J. Pineal region surgery. Experience in 22 patients. Neurochirurgie. 2002;48:14-24.

9. Hellwig D, Bauer BL, List-Hellwig E. Stereotactic endoscopic interventions in cystic brain lesions. Acta Neurochir Suppl. 1995;64:59-63.

10. Gaab MR, Schroeder HW. Neuroendoscopic approach to intraventricular lesions. Neurosurg Focus. 1999;6:5. 